\title{
Relationship Between Periosteal Reaction and Medial Compartment Knee Osteoarthritis in Observation of Chinese Patients: A Retrospective Single-Centre Comparative Study
}

\author{
Xiangtian Deng \\ Nankai University School of Medicine \\ Hongzhi Hu \\ Huazhong University of Science and Technology \\ Xiaodong Cheng \\ Hebei Medical University Third Affiliated Hospital \\ Jian Zhu \\ Nankai University School of Medicine \\ Zhipeng Ye \\ Nankai University School of Medicine \\ Zhongzheng Wang \\ Hebei Medical University Third Affiliated Hospital \\ Yuchuan Wang \\ Hebei Medical University Third Affiliated Hospital \\ Yingze Zhang ( $\nabla$ zyz050051@163.com ) \\ Hebei Medical University Third Affiliated Hospital
}

Research article

Keywords: Knee osteoarthritis, Medial compartment, Periosteal reaction, proximal tibial shaft

Posted Date: September 15th, 2020

DOI: https://doi.org/10.21203/rs.3.rs-74153/v1

License: (c) (i) This work is licensed under a Creative Commons Attribution 4.0 International License. Read Full License 


\section{Abstract}

Background:The purpose of this study to evaluate the potential relationship between the periosteal reaction and medial compartment knee osteoarthritis (KOA), and to assess the related risk factors for the development of periosteal reaction associated with medial compartment KOA.

Methods: From January 2019 to December 2019, a total of 476 consecutive patients with medial compartment KOA were included and assessed in this retrospective comparative study. They were divided into two groups: periosteal reaction group and non-periosteal reaction group. Radiological parameters was measured for malalignment of the lower extremities in coronal plane. Intra-observer and interobserver reliabilities of all radiological measurements were analyzed by intraclass correlation coefficients (ICCs). Univariate analyses was conducted for comparison of differences with continuous variables between patients with periosteal reaction and without periosteal reaction. Multivariate binary logistical regression analysis were performed to determine the independent risk factors of radiographic parameters for periosteal reaction.

Results: A total of 363 patients (726 knees) were selected for the study, including 91 males and 272 females, with an average age of $57.9 \pm 12.8$ years (range, $18-82$ years). The overall incidence of periosteal reaction associated with medial compartment KOA was $56.7 \%$. Furthermore, the incidence of periosteal reaction significantly increased with age and K-L grade progressed ( $P \otimes 0.05)$. Multivariate logistical regression analysis found that HKA and JICA were significantly correlated with the development of periosteal reaction adjacent to the lateral of proximal tibia diaphysis in medial compartment KOA $(P]$ $0.05)$.

Conclusions: Our observation of the reaction of periosteum may be an anatomical adaptation for medial compartment KOA. Patients with lower HKA and higher JICA are more likely to developing periosteal reaction, which occurred most commonly adjacent to the lateral of proximal tibia diaphysis.

\section{Background}

Knee osteoarthritis (KOA) is one of the most common joint disorders and often increases the risk of disability for patients, which has the highest incidence rate between the ages of 55-65 years and poses an enormous socioeconomic burden for the worldwide [1, 2]. The common symptoms in patients with medial compartment KOA contains pain and limited range of motion, which ultimately result in malalignment of the lower extremities and intra-articular cartilage wear [3]. Furthermore, disproportionate of the loading into the knee joint with the varus malalignment make contribution to the KOA progression [4].

Periosteum, located at the interface between the cortex and peri-osseous soft tissues, is a thin but tough peripheral membrane of the bone which contains fibrous layer and cambial layer, and plays an important role in bone remodeling for adults [5]. Although periosteum exhibit several crucial effects in children and adults, orthopedists have pay little attention to this dynamic structure. Periosteal reaction in plain 
radiography can be seen as different thickness lines which may closely parallel to the cortex of the bone. Some authors have proven that there could be a wide variety of reasons induce periosteal bone formation, including trauma, infection, metabolic diseases, inflammatory disorders, systemic diseases, benign and malignant tumors [6-9]. At mean time, during the occurrence and progression of medial compartment KOA, we found a general phenomenon that the development of periosteal reaction is often occurred in lateral of proximal tibia diaphysis according to the standing anteroposterior (AP) radiographs.

Unfortunately, little is known about the characteristics of cortical layer of the surface changes associated with KOA, nor the radiological measurements of these changes in relationship with coronal malalignment. According to our clinical observation and experience, we believed that the non-uniform settlement of tibial plateau would result in the increase of compressive forces on the medial side of proximal tibia shaft while the tensile stress increased on the lateral side of proximal tibia shaft, which may in turn stimulates periosteum to produce new bone on the cortical layer of the surface of the tibia. Consequently, we proposed for the first time the concept of "adaptive periosteal reaction" (APR) in response to this phenomenon in this study.

To the best of our knowledge, there has been no study that investigates APR adjacent to the lateral of proximal tibia diaphysis in patients with medial compartment KOA. Herein, a consecutive cohort of patients with medial compartment KOA presented in our hospital was retrospective analyzed. The purpose of the present study was to evaluate the potential relationship between the periosteal reaction and medial compartment KOA, and to make further exploration on the measurement of radiographic parameters correlated with periosteal reaction in patients with medial compartment KOA.

\section{Methods}

This study was a single-center retrospective comparative study and was approved by the Ethical Committee of third hospital of Hebei Medical University and was conducted in accordance with the Declaration of Helsinki. All individual participants provided written informed consents to their participation in this study. Weight-bearing in full limb radiographs obtained in our hospital from January 2019 to December 2019 were reviewed through the picture archiving and communication systems (PACS; Science \& Technology General Company of Hebei Medical University, Shijiazhuang, China).

\section{Inclusion criteria and exclusion criteria}

Patients meeting the following criteria were included: a) age $\geq 40$ years; b) participants diagnosed with medial compartment KOA based on American Rheumatism Association clinical and radiographic criteria [10]; c) patients in standard weight-bearing of long-standing AP views (both the patella and feet facing forwards). The exclusion criteria were as follows: a) incomplete demographic data; b) history of prior knee surgery; c) post-traumatic or inflammatory arthritis; d) patellofemoral joint and lateral compartment osteoarthritis; e) lower extremities with plain radiographs in the position of internal or external rotation.

\section{Radiological assessments}


The radiographic severity of KOA was evaluated by the radiographic classification systems such as the Kellgren and Lawrence (K-L) classification [11]. Fig.1 demonstrating periosteal reaction in the lateral of proximal tibia diaphysis in medial compartment KOA. The medial proximal tibial angle (MPTA) was defined the medial angle between the tibial mechanical axis and the tangent line of the medial and lateral edges of the tibial plateau, indicating the alignment of tibia on coronal plane [12]. Moreover, the hip-kneeankle angle (HKA) of the lower extremity was the angle calculated by intersecting the line between femoral mechanical axis (from the center of the femoral head to the central point of the tibial spines) and tibial mechanical axis (from the center of the tibial spines to the center of the superior articular surface of the talus), indicating the alignment of the lower extremity [13]. The lateral distal femoral angle (LDFA), defined as the angle between the tangent to the distal femoral condyle line and the femoral mechanical axis, representing the alignment of femoral on coronal plane [14]. The joint line convergence angle (JICA) was formed by two articular tangential line of the distal femur and proximal tibia, evaluating intraarticular cartilage loss and soft tissue laxity on the coronal plane [15]. The minimum medial joint space width (min-JSW) was the minimum distance of medial knee joints (Fig.3). All patients in radiological parameters were assessed by two senior radiologists, independently of each other, to measure the K-L classification, MPTA, HKA, LDFA, JICA, and min-JSW. One observer repeated the measurements with an interval of two weeks. The average of the radiological measurements was recorded and used for analyses.

\section{Statistical analysis}

SPSS software (version 25.0, IBM Corp., USA) was performed for statistical analysis. Intraclass correlation coefficients (ICCs) were used to assess intra-observer and inter-observer reliability in evaluating radiological parameters. Univariate analyses was conducted for comparison of differences with continuous variables between patients with periosteal reaction and without periosteal reaction, Chisquare tests were used to compare the difference for categorical variables. Multivariate binary logistical regression analysis were performed to determine the independent risk factors of radiographic parameters for periosteal reaction.

\section{Results}

In this study, a total of 476 consecutive patients with long-standing anterior-posterior (AP) radiographs were identified in the PACS system during this time. Out of 476 patients, 113 patients were excluded according to our inclusion criteria, and 363 patients (726 knees) were selected for the study, including 91 males and 272 females, with an average age of $57.9 \pm 12.8$ years (range, 18-82 years). Of the 726 knees in this series, two hundred and six patients (412 knees, 56.7\%) were classified into APR group, and 157 patients (314 knees, 45.3\%) were classified as non-APR group. From the univariate analyses, there is significant differences for gender (male/female, 44/162 vs 47/110) when comparing the APR group with the non-APR group ( $P \otimes 0.05)$, which suggests that females have higher incidence of periosteal reaction than males. In the APR group, the mean MPTA, HKA, LDFA, JICA, and min-JSW were $82.3^{\circ} \pm 2.6^{\circ}, 170.8^{\circ}$ $\pm 4.9^{\circ}, 88.2^{\circ} \pm 2.8^{\circ}, 4.2^{\circ} \pm 1.9^{\circ}$, and $2.6 \pm 1.8 \mathrm{~mm}$, respectively. In the non-APR group, the mean MPTA, HKA, 
LDFA, JICA, and min-JSW were $84.3^{\circ} \pm 3.8^{\circ}, 176.7^{\circ} \pm 3.1^{\circ}, 88.6^{\circ} \pm 2.7^{\circ}, 3.1^{\circ} \pm 2.2^{\circ}$, and $3.8 \pm 1.9 \mathrm{~mm}$, respectively. The MPTA, HKA, JICA, and min-JSW were significantly lower in the APR group compared to the non-APR group ( $P \bowtie 0.05)$. In contrast, there were no significant difference on LDFA between the two groups $(P \llbracket 0.05)$. Demographic data and radiological measurements were comparable, as shown in Table 1. 
Table 1

Comparison of patient demographics and radiographic parameters between the subgroups

\begin{tabular}{|c|c|c|c|}
\hline Parameters & APR group & Non-APR group & $P$ value \\
\hline Number of knees & $412 / 726(56.7 \%)$ & $314 / 726$ (43.3\%) & $\mathrm{N} / \mathrm{A}$ \\
\hline Age (years) & & & $<0.001^{*}$ \\
\hline$\nabla 40$ & 32/91 (35.2\%) & $59 / 91(64.8 \%)$ & \\
\hline $40-60$ & $142 / 272(52.2 \%)$ & $130 / 272(47.8 \%)$ & \\
\hline$\geq 60$ & $238 / 363(65.6 \%)$ & $125 / 363$ (34.4\%) & \\
\hline Gender (\%) & & & $<0.001^{*}$ \\
\hline Male & 44/91 (48.4\%) & 47/91 (51.6\%) & \\
\hline Female & $162 / 272(59.6 \%)$ & $110 / 272(40.4 \%)$ & \\
\hline K-L classification (\%) & & & $<0.001^{*}$ \\
\hline Grade I & $20 / 100(20.0 \%)$ & $80 / 100(80.0 \%)$ & \\
\hline Grade II & $83 / 182(45.6 \%)$ & 99/182 (54.4\%) & \\
\hline Grade III & 208/312 (66.7\%) & 104/312 (33.3\%) & \\
\hline Grade IV & 101/132 (76.5\%) & $31 / 132(23.5 \%)$ & \\
\hline $\operatorname{MPTA}\left({ }^{\circ}\right)$ & $82.3^{\circ} \pm 2.6^{\circ}$ & $84.3^{\circ} \pm 3.8^{\circ}$ & $<0.001^{*}$ \\
\hline $\operatorname{HKA}\left({ }^{\circ}\right)$ & $170.8^{\circ} \pm 4.9^{\circ}$ & $176.7^{\circ} \pm 3.1^{\circ}$ & $<0.001^{*}$ \\
\hline $\operatorname{LDFA}\left({ }^{\circ}\right)$ & $88.2^{\circ} \pm 2.8^{\circ}$ & $88.6^{\circ} \pm 2.7^{\circ}$ & 0.899 \\
\hline $\operatorname{JICA}\left({ }^{\circ}\right)$ & $4.2^{\circ} \pm 1.9^{\circ}$ & $3.1^{\circ} \pm 2.2^{\circ}$ & $<0.001^{*}$ \\
\hline Min-JSW (mm) & $2.6 \pm 1.8$ & $3.8 \pm 1.9$ & $<0.001^{*}$ \\
\hline \multicolumn{4}{|c|}{$\begin{array}{l}\text { Continuous variables were presented as mean } \pm \text { standard deviation, while categorical variables wer } \\
\text { presented as number or percentage. }\end{array}$} \\
\hline \multicolumn{4}{|c|}{$\begin{array}{l}N / A \text { not available, } A P R \text { adaptive periosteal reaction, } K-L \text { Kellgren and Lawrence, MPTA medial } \\
\text { proximal tibial angle, HKA hip-knee-ankle angle, } L D F A \text { lateral distal femoral angle, JICA joint line } \\
\text { convergence angle, min-JSW minimum joint space width }\end{array}$} \\
\hline
\end{tabular}

In addition, our results also showed that significant differences in the incidence of periosteal reaction among different age groups and K-L grades. The distribution of the 726 knees based on $\mathrm{K}-\mathrm{L}$ classification was as follows: K-L grade I were 100 knees (13.8\%). K-L grade II were 182 knees (25.1\%). K-L grade III were 312 knees (43.0\%). K-L grade IV were 132 knees (18.2\%). Table 1 demonstrated the specific 
information regarding incidence of periosteal reaction based on each K-L classification and different age groups, which showed a significant tendency to increase with age and K-L grade. Intra-observer and interobserver reliability assessment of two groups for radiological parameters are shown in Table 2. All ICCs values of radiological measurements for intra-observer and inter-observer reliability were exceeded 0.8 , indicating excellent agreement.

Table 2

Intra-observer and inter-observer reliability assessment of two groups for radiological parameters

\begin{tabular}{|llllll|}
\hline \multirow{2}{*}{ Measurements } & \multicolumn{2}{l}{ Intra-observer } & & \multicolumn{2}{l|}{ Inter-observer } \\
\cline { 2 - 3 } \cline { 5 - 6 } & ICC & $95 \% \mathrm{Cl}$ & & ICC & $95 \% \mathrm{Cl}$ \\
\hline K-L classification & 0.946 & $0.912-0.974$ & & 0.938 & $0.907-0.967$ \\
\hline MPTA $\left(^{\circ}\right)$ & 0.932 & $0.832-0.968$ & & 0.927 & $0.898-0.954$ \\
\hline HKA $\left(^{\circ}\right)$ & 0.911 & $0.847-0.956$ & 0.909 & $0.854-0.949$ \\
\hline LDFA $\left(^{\circ}\right)$ & 0.921 & $0.843-0.961$ & & 0.914 & $0.867-0.948$ \\
\hline JICA $\left({ }^{\circ}\right)$ & 0.878 & $0.768-0.921$ & 0.861 & $0.752-0.906$ \\
\hline Min-JSW (mm) & 0.862 & $0.757-0.919$ & 0.874 & $0.751-0.901$ \\
\hline ICC intraclass correlation coefficient, Cl confidence interval \\
\hline
\end{tabular}

Multivariate binary logistic regression was performed to analyze the correlation of radiological parameters with APR, indicating that HKA and JICA were significantly negatively correlated with the development of APR (respectively, odds ratio OR $=0.594,95 \% \mathrm{Cl}=0.544-0.648 ; \mathrm{OR}=0.851,95 \% \mathrm{Cl}=$ 0.737-0.983). Meanwhile, JICA had the highest correlation with the risk of APR, and the differences were statistically significant $(P \otimes 0.05)$. These results demonstrated that patients with lower HKA and higher JICA were susceptible to periosteal reaction. In contrast, MPTA and LDFA were negatively correlated with periosteal reaction risk (respectively, $\mathrm{OR}=0.959,95 \% \mathrm{Cl}=0.901-1.021 ; \mathrm{OR}=0.990,95 \% \mathrm{Cl}=0.865-1.167$, $P \otimes 0.05)$, but the difference was not statistically significant (P凶0.05), as shown in Table 3. 
Table 3

Multivariate binary logistic regression analysis of risk factors in radiological parameters associated with periosteal reaction in medial compartment KOA

\begin{tabular}{|lll|}
\hline Variables & OR and 95\% Cl & $P$ \\
\hline MPTA $\left(^{\circ}\right)$ & $0.959(0.901-1.021)$ & 0.193 \\
\hline HKA $\left(^{\circ}\right)$ & $0.594(0.544-0.648)$ & $<0.001^{*}$ \\
\hline LDFA $\left(^{\circ}\right)$ & $0.990(0.899-1.089)$ & 0.831 \\
\hline JICA $\left(^{\circ}\right)$ & $0.851(0.737-0.983)$ & $0.029 *$ \\
\hline Min-JSW (mm) & $1.005(0.865-1.167)$ & 0.951 \\
\hline $\begin{array}{l}\text { Abbreviation and notes: MPTA medial proximal tibial angle, HKA hip-knee-ankle angle, LDFA lateral } \\
\text { distal femoral angle, JiCA joint line convergence angle, min-JSW minimum joint space width }\end{array}$ & \\
\hline *Significant variables & & \\
\hline
\end{tabular}

\section{Discussion}

The most important finding of the present study was that the development of periosteal reaction in lateral of proximal tibia diaphysis is a radiographic finding associated with medial compartment KOA, and showed that HKA and JICA were demonstrated to be independent risk factors associated with the occurrence of periosteal reaction rather than MPTA, LDFA and min-JSW as KOA grades progressed.

To date, the present study is the first to evaluate the relationship between the periosteal reaction and medial compartment knee osteoarthritis (KOA), and to assess the independent risk factors for the presence of periosteal reaction. The results of this study identified that the prevalence of APR significantly increased as K-L grades progressed in patients with medial compartment KOA. In the present study, our results also showed that with the increased incidence of APR, the medial proximal tibial angle became lower, and the joint line convergence angle became larger, which lead to varus malalignment of lower extremity ultimately. This finding of our study was consistent with our hypothesis, showing that the cortical layer changes of the periosteum may be an adaptation to the progression of medial compartment KOA, which could be one of the mechanisms for development of KOA. Dong et al. [16] have shown that non-uniform settlement of tibial plateau was closely related to the varus malalignment in medial compartment KOA. Increased joint loading in the medial compartment and occurrence of osteoporosis with age can increase the risk of bone trabecula thinner and bone loss. It has been reported that the cambial layer situated adjacent to the cortex bone contains periosteum derived stem cells (PDCs) that are sensitive to inflammatory syndrome and mechanical stimulation [17]. In addition, Moore et al. [18] showed that PDCs can be recruited to fibrous layer and modulated by mechanical stress. Based on these previous literatures and our results, therefore, we considered that when the excessive axial load-bearing distributed to the medial side of proximal tibia diaphysis, distraction osteogenesis of the lateral side of proximal tibia would occur due to the imbalance mechanically environment of the proximal tibia shaft. 
In the present study, we also investigated whether radiological parameters in coronal plane are correlated with APR in patients with medial compartment KOA. Multivariate binary logistic regression analysis identified that HKA and JICA were independent risk factors for the development of APR (OR $=0.594$ and 0.851 , respectively). This may be partially explained by the theory of non-uniform settlement of tibial plateau. More recently, our published studies reported that the lateral curvature of the proximal fibular concomitant with aging may be an anatomical adaptation for medial compartment KOA, and these changes in the fibula was in correlation with settlement of the medial plateau [19]. Similarly, based on the findings of this study, we assume that the lateral side of the proximal tibia shaft redistributes the excessive loading to the periosteum, while this increased tensile stress could indirectly affect cortical layer of the surface changes as well. Furthermore, among varus malalignment factors, JICA was the most significant factor in association with the presence of periosteal reaction. Nakayama et al [20] have described that larger JICA may induce shear stress on articular cartilage. This increased shear stress that transmitted to proximal tibia may indirectly affect the occurrence of periosteal reaction. Nevertheless, no comparable studies reporting on periosteal reaction in association with medial compartment KOA.

In this research, we applied MPTA and LDFA to evaluate the coronal malalignment of the lower limb instead of using HKA alone due to both the tibia and femur varus deformities are associated with varus malalignment [21, 22]. It has been suggest that periosteal reaction is believed to be the result from regional certain mechanical factors [23]. This anatomical adaptation of the tibia was in relation with excessive tensile stress in periosteum due to the long-term bearing in lateral side of proximal tibial shaft. A clear understanding of correlation with radiological measurements for APR occurrence may help orthopaedic surgeons develop further insight into the natural history of KOA and the mechanisms of medial compartment KOA. Furthermore, based on this background, the adaptive changes of the periosteum in proximal tibia shaft may further illuminates the theory of non-uniform settlement of tibial plateau on medial compartment KOA.

However, there are some noteworthy limitations to this retrospective comparative study. First, we did not perform the histopathological observation for the development of APR in medial compartment KOA. Second, the thickness of the periosteal reaction was not determined quantitatively, and body mass index (BMI) could not be assessed for each patient in this study. Third, all the measurements were evaluated on coronal plane of the lower extremities and did not include sagittal plane. Furthermore, the relatively small sample patients may have bias for analysis of our results. Therefore, further studies with large-scale prospective study are still needed to focus on exploring the underlying mechanisms of APR and its role in the development of medial compartment KOA.

\section{Conclusions}

In conclusion, this is the first study on the relationship between the occurrence of periosteal reaction to the lateral of proximal tibial shaft and medial compartment KOA in Chinese patients. Our finding suggests that the periosteal reaction is a radiographic finding related to medial compartment KOA and the incidence of periosteal reaction significantly increased with age and K-L grades progressed. 
Furthermore, patients with lower HKA and higher JICA are more likely to developing periosteal reaction which occurred most commonly adjacent to the lateral of proximal tibia shaft.

\section{Abbreviations}

APR: adaptive periosteal reaction

K-L: Kellgren and Lawrence,

MPTA: medial proximal tibial angle

HKA: hip-knee-ankle angle

LDFA: lateral distal femoral angle

JICA: joint line convergence angle

min-JSW: minimum joint space width

\section{Declarations}

\section{Availability of data and materials}

All the data and material involving this article will be available upon request by send an e-mail to the first author.

\section{Acknowledgements}

We are grateful to the $\mathrm{J}$ Wang and $\mathrm{W}$ Chen of the Department of Orthopedics for their kind assistance.

\section{Funding}

This study was supported by the National Natural Science Foundation of China (Grant No. 81401789).

\section{Author's contributions}

YZZ designed the study; WY ,WZ, YZ, and ZJ searched relevant studies; XTD and HZH analyzed and interpreted the data; XTD and XDC wrote the manuscript; and YZZ approved the final version of the manuscript.

\section{Ethics declarations}

\section{Ethics approval and consent to participate}

This study was approved by the ethics committee of the $3^{\text {rd }}$ Hospital of Hebei Medical University. Informed consent was obtained from all individual participants included in the study. 


\section{Consent for publication}

Consent to publish was obtained from each patient.

\section{Competing interests}

All the authors declare that they have no conflict of interest with any organization.

\section{References}

1. Cross M, Smith E, Hoy D. et al(2014)The global burden of hip and knee osteoarthritis: estimates from the global burden of disease 2010 study. ANN RHEUM DIS. 73(7): 1323-1330. doi: 10.1136/annrheumdis-2013-204763.

2. Driban JB, McAlindon TE, Amin M. et al(2018)Risk factors can classify individuals who develop accelerated knee osteoarthritis: Data from the osteoarthritis initiative. J ORTHOP RES. 36(3): 876880. doi: 10.1002/jor.23675.

3. Sharma L, Song J, Felson DT. et al(2001)The role of knee alignment in disease progression and functional decline in knee osteoarthritis. JAMA. 286(2): 188-195. doi: 10.1001/jama.286.2.188.

4. Hayashi D, Englund M, Roemer FW, et al(2012)Knee malalignment is associated with an increased risk for incident and enlarging bone marrow lesions in the more loaded compartments: the MOST study. Osteoarthritis Cartilage. 20(11): 1227-1233. doi: 10.1016/j.joca.2012.07.020.

5. Dwek JR. The periosteum: what is it, where is it, and what mimics it in its absence? SKELETAL RADIOL. 2010;39(4):319-23. doi:10.1007/s00256-009-0849-9.

6. Meijers KA, Pare DM, Loose $\mathrm{H}$, et al(1982)Periarteritis nodosa and subperiosteal new bone formation. J Bone Joint Surg Br. 64(5): 592-596. doi.

7. Zeng X, Huang H, Li J, et al(2018)The Clinical and Radiological Characteristics of Inflammatory Myofibroblastic Tumor Occurring at Unusual Sites. BIOMED RES INT. 2018: 5679634. doi:10.1155/2018/5679634.

8. Major NM, Crawford S. T(2002)Elbow effusions in trauma in adults and children: is there an occult fracture? AJR Am J Roentgenol. 178(2): 413-418. doi: 10.2214/ajr.178.2.1780413.

9. Ragsdale BD, Madewell JE, Sweet DE. Radiologic and pathologic analysis of solitary bone lesions. Part II: periosteal reactions. Radiol Clin North Am. 1981;19(4):749-83. doi.

10. Altman R, Asch E, Bloch D. et al(1986)Development of criteria for the classification and reporting of osteoarthritis. Classification of osteoarthritis of the knee. Diagnostic and Therapeutic Criteria Committee of the American Rheumatism Association. Arthritis Rheum. 29(8): 1039-1049. doi: $10.1002 /$ art.1780290816.

11. KELLGREN J H, LAWRENCE J S(. Radiological assessment of osteo-arthrosis. ANN RHEUM DIS. 1957;16(4):494-502. doi:10.1136/ard.16.4.494. 
12. Haviv B, Bronak S, Thein R. et al(2013)The results of corrective osteotomy for valgus arthritic knees. Knee Surg Sports Traumatol Arthrosc. 21(1): 49-56. doi: 10.1007/s00167-012-2180-6.

13. Cho MR, Lee YS, Choi WK(2018)Relationship between Lateral Femoral Bowing and Varus Knee Deformity Based on Two-Dimensional Assessment of Side-to-Side Differences. Knee Surg Relat Res. 30(1): 58-63. doi: 10.5792/ksrr.17.007.

14. Nakano N, Matsumoto $T$, Hashimura $M$, et al(2016)Coronal lower limb alignment in normal knees-A radiographic analysis of 797 normal knee subjects. KNEE. 23(2): 209-213. doi: 10.1016/j.knee.2015.12.004.

15. Lee DH, Park SC, Park HJ. et al(2016)Effect of soft tissue laxity of the knee joint on limb alignment correction in open-wedge high tibial osteotomy. Knee Surg Sports Traumatol Arthrosc. 24(12): 37043712. doi: 10.1007/s00167-015-3682-9.

16. Dong T, Chen W, Zhang F. et al(2016)Radiographic measures of settlement phenomenon in patients with medial compartment knee osteoarthritis. CLIN RHEUMATOL. 35(6): 1573-1578. doi: 10.1007/s10067-015-3146-0.

17. Colnot $C$, Zhang $X$, Knothe T. $M(2012)$ Current insights on the regenerative potential of the periosteum: molecular, cellular, and endogenous engineering approaches. J ORTHOP RES. 30(12): 1869-1878. doi: 10.1002/jor.22181.

18. Moore SR, Milz S, Knothe T. M(2014)Periosteal thickness and cellularity in mid-diaphyseal crosssections from human femora and tibiae of aged donors. J ANAT. 224(2): 142-149. doi: 10.1111/joa.12133.

19. Wang J, Lv HZ, Chen W. et al(2019)Anatomical Adaptation of Fibula and its Mechanism of Proximal Partial Fibulectomy Associated with Medial Compartment Knee Osteoarthritis. ORTHOP SURG. 11(2): 204-211. doi: 10.1111/os.12437.

20. Nakayama H, Schroter S, Yamamoto C. et al(2018)Large correction in opening wedge high tibial osteotomy with resultant joint-line obliquity induces excessive shear stress on the articular cartilage. Knee Surg Sports Traumatol Arthrosc. 26(6): 1873-1878. doi: 10.1007/s00167-017-4680-x.

21. Hirschmann MT, Moser LB, Amsler F, et al(2019)Phenotyping the knee in young non-osteoarthritic knees shows a wide distribution of femoral and tibial coronal alignment. Knee Surg Sports Traumatol Arthrosc. 27(5): 1385-1393. doi: 10.1007/s00167-019-05508-0.

22. Bonnin MP, Saffarini M, Bossard N. et al(2016)Morphometric analysis of the distal femur in total knee arthroplasty and native knees. BONE JOINT J. 98-B(1): 49-57. doi: 10.1302/0301620X.98B1.35692.

23. Bisseret $\mathrm{D}$, Kaci $\mathrm{R}$, Lafage-Proust $\mathrm{MH}$, et al(2015)Periosteum: characteristic imaging findings with emphasis on radiologic-pathologic comparisons. SKELETAL RADIOL. 44(3): 321-338. doi: 10.1007/s00256-014-1976-5.

\section{Figures}




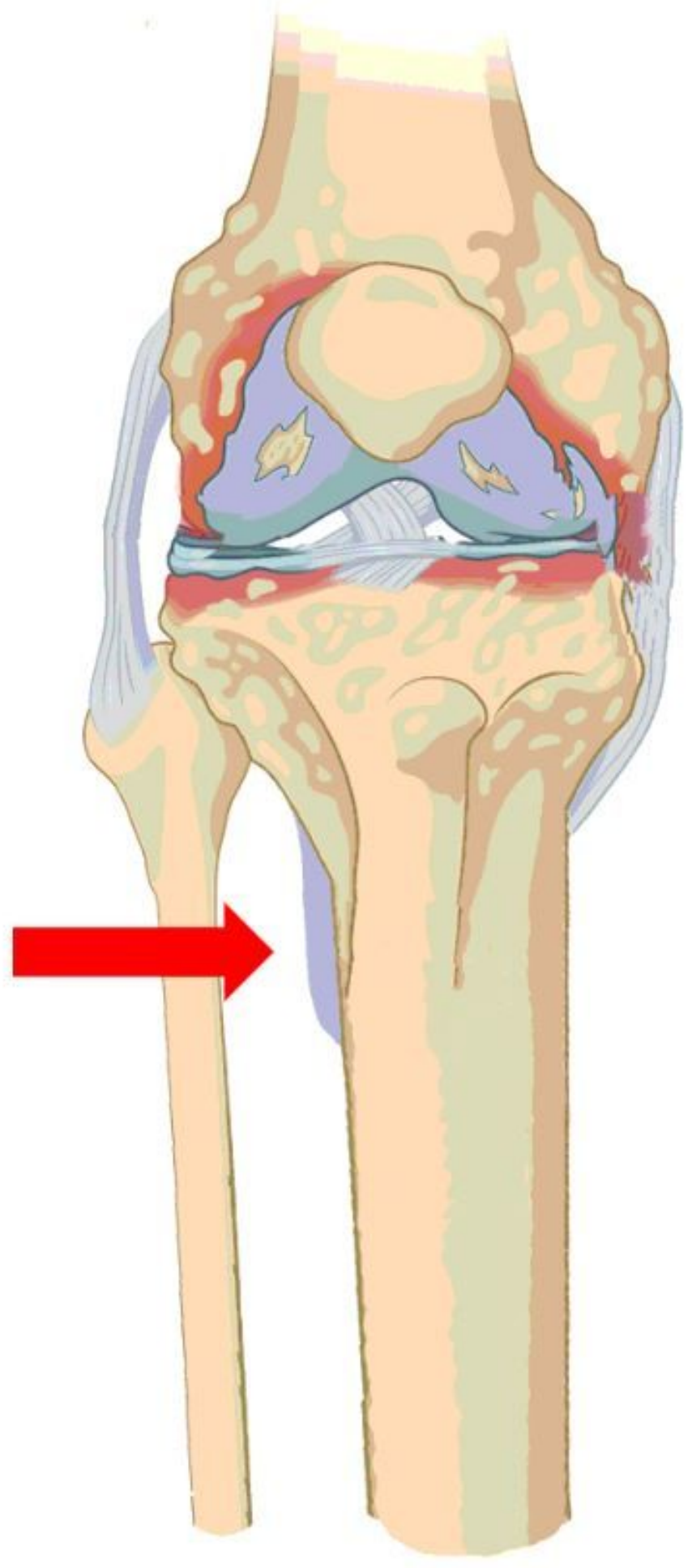

Figure 1

Schematic illustration demonstrating periosteal reaction (red arrow) in the lateral of proximal tibia diaphysis in medial compartment KOA. 


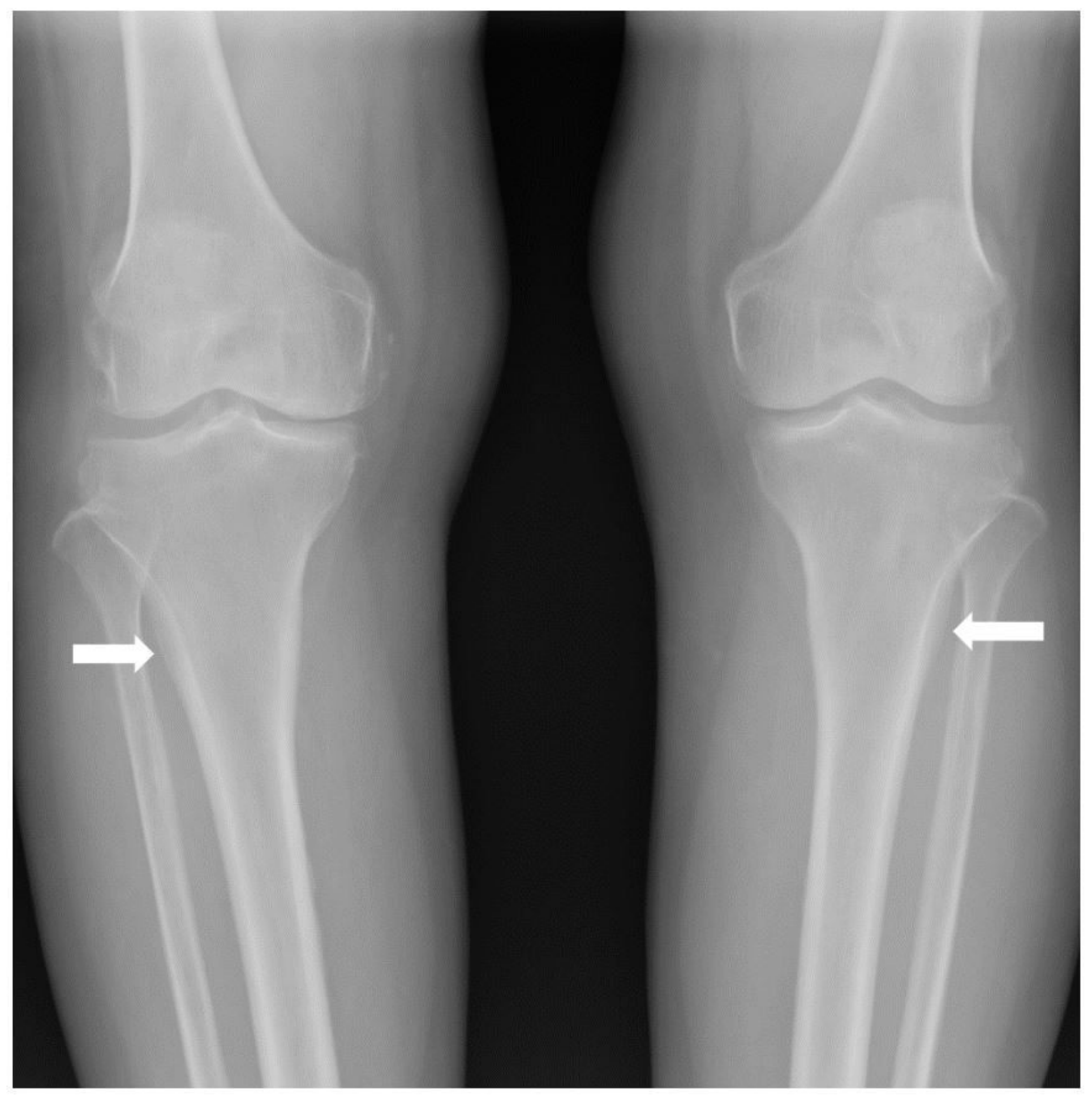

\section{Figure 2}

Periosteal reaction in patient with medial compartment KOA in both knees. Weight-bearing knee AP radiographs demonstrated regional periosteal reaction (arrow) in both of the lateral proximal tibia shaft. 


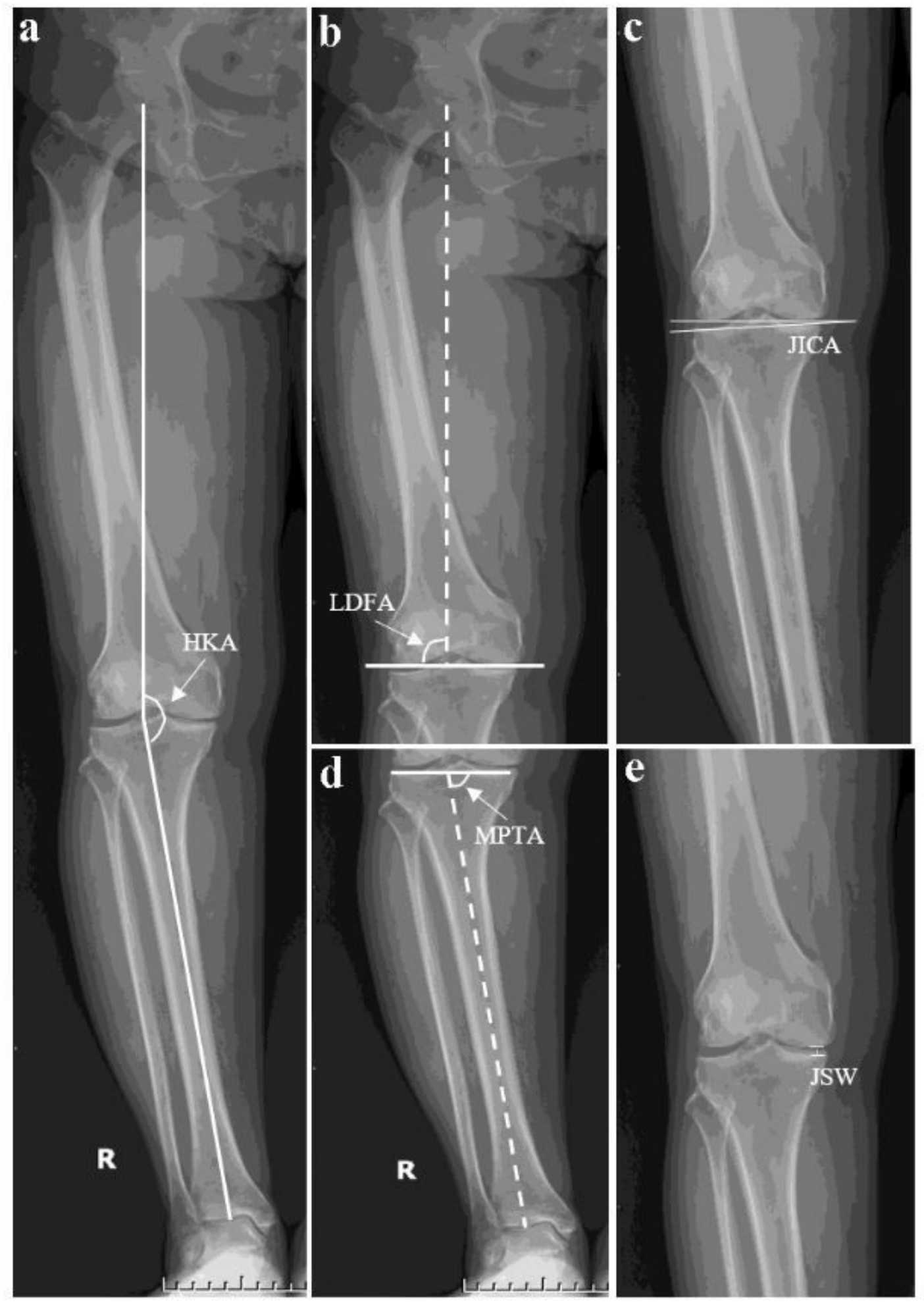

\section{Figure 3}

Radiological measurements of the lower extremity in coronal plane. a Hip-knee-ankle (HKA) was the angle calculated by intersecting the line between femoral mechanical axis (from the center of the femoral head to the central point of the tibial spines) and tibial mechanical axis (from the center of the tibial spines to the center of the superior articular surface of the talus. $b$ Lateral distal femoral angle (LDFA) was the angle between the tangent to the distal femoral condyle line and the femoral mechanical axis. c Medial 
proximal tibial angle (MPTA) was defined as the medial angle between the mechanical axis of the tibia and the proximal tibial articular axis. $d$ Joint line convergence angle (JICA) was formed by two articular tangential line of the distal femur and proximal tibia. e Joint space width (JSW) was the minimum distance of knee joints. 